\title{
Critical Comments on the Paper "On the Logical Inconsistency of the Special Theory of Relativity"
}

\author{
Vladimir A. Leus \\ Sobolev Institute of Mathematics, Novosibirsk, Russia \\ Email: v.leus@liverpool.ac.uk
}

How to cite this paper: Leus, V.A. (2018) Critical Comments on the Paper "On the Logical Inconsistency of the Special Theory of Relativity". Journal of Applied Mathematics and Physics, 6, 925-931. https://doi.org/10.4236/jamp.2018.64079

Received: February 22, 2018

Accepted: April 27, 2018

Published: April 30, 2018

Copyright $\odot 2018$ by author and Scientific Research Publishing Inc. This work is licensed under the Creative Commons Attribution International License (CC BY 4.0).

http://creativecommons.org/licenses/by/4.0/

\section{cc) (i) Open Access}

\begin{abstract}
Special Relativity Theory is more than 110 years aged and during this period it was elaborated until minuscule details. However, there might be some logically deduced discrepancies, which demand a scrupulous study. Nonetheless, every search for inherent contradictions is an uphill task. The author of the considered paper proposed a situation with two series of synchronized clocks. Each series is at rest in its own frame of reference, but one of them is deemed to be stationary and other is moving with a constant relative velocity. The author believes this situation to be contradictable. But really, the suitable mathematical analysis proves that it is none other than a consequence of neglecting the basic tenets of the theory.
\end{abstract}

\section{Keywords}

Special Relativity Theory, Relativity of Simultaneity, Pseudo-Contradictions

\section{About Time Dilation}

There are two inertial frames of reference $I F R_{x}$ - stationary, and $I F R_{\xi}$ -moving with a relative constant velocity $\boldsymbol{v}=(v, 0,0)$, where the component $v$ is less than speed of light $c$ (the author names them briefly system $K$ and system $k$ ). Both abscissa-axis $x$ and $\xi$ are in parallel, and both coordinate origins coincide at the zero moment of time $t_{0}=\tau_{0}=0$. A series of real numbers $\sigma_{i}$ is fixed, the nil $\sigma_{0}=0$ being amongst them. Starting from an arbitral abscissa $x_{1} \neq 0$ a range of positions on the $x$-axis is set according to the rule $x_{\sigma}=\sigma x_{1}$, where $\sigma$ denotes a number $\sigma_{i}$ from the series (Figure 1).

In the second section of his paper [1] the author considers a range of events occurring at positions $x_{\sigma_{i}}=x_{i}$ in different moments of time $t_{i}$, which are 


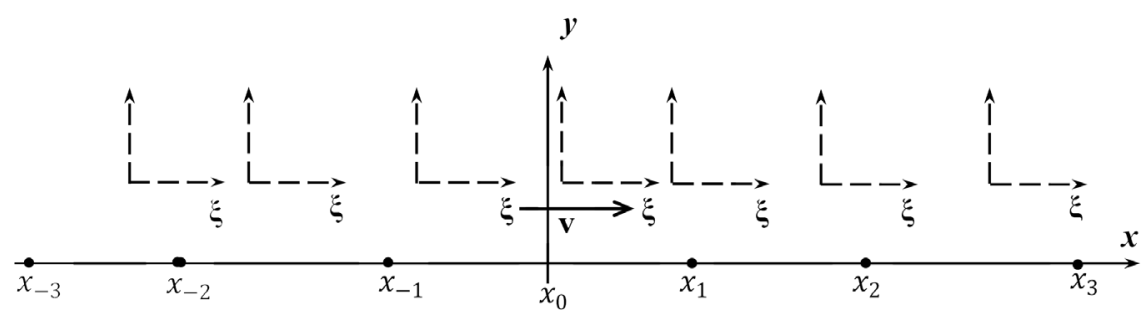

Figure 1. Stationary and moving IFRs in different moments of time.

specified as follows:

$$
t_{\sigma_{i}}=t_{i}=t_{1}+\frac{\left(\sigma_{i}-1\right) v x_{1}}{c^{2}}
$$

where $t_{1}$ is at will. In this paper we read: "The Lorentz Transformation is the basis for Einstein's time dilation and length contraction. It is regarded in general by physicists that a stationary system of observers $k$ which are clock-synchronized when at rest are not synchronized when they all move together with respect to a clock-synchronized 'stationary system' $K$, as illustrated in figure 1'.

The depicted drawing is rather bewildering than helpful. Nothing similar can be going on if the order established in the special relativity is strictly kept. A correct illustration is delineated in Figure 2. In the $k$ system a clock vis-à-vis to a clock in the $K$ system is $\gamma$ times more distant from the $k$ origin than the second one is from the $K$ origin. Here the relativistic factor $\gamma=\left(1-\frac{v^{2}}{c^{2}}\right)^{-1 / 2}$, where $c$ is the light speed in vacuum [2]. All the clocks in the $k$ system remain synchronized despite its uniform motion with respect to the "stationary system" $K$.

The author uses the Lorentz transformation equations

$$
\xi_{\sigma}=\gamma\left(x_{\sigma}-v t_{\sigma}\right), \tau_{\sigma}=\gamma\left(t_{\sigma}-\frac{v}{c^{2}} x_{\sigma}\right)
$$

in order to obtain positions $\xi_{\sigma}$ and moments $\tau_{\sigma}$ in the $k$ system correspondent to positions $x_{\sigma}$ and moments $t_{\sigma}$ in the $K$ system. They are as follows:

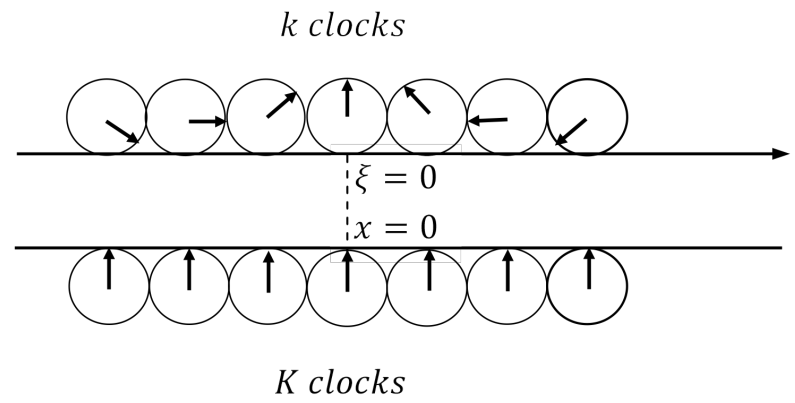

figure 1. All the synchronized clocks in the "stationary system" $K$ read the same time at all positions in the $K$ system. All the clocks in the "moving system" $k$ do not read the same time according to the $K$ system, despite being synchronized with respect to the $k$ system. Only at $x=\xi=0$ do the clocks depicted read the same time in both systems, where $t=\tau=0$. 


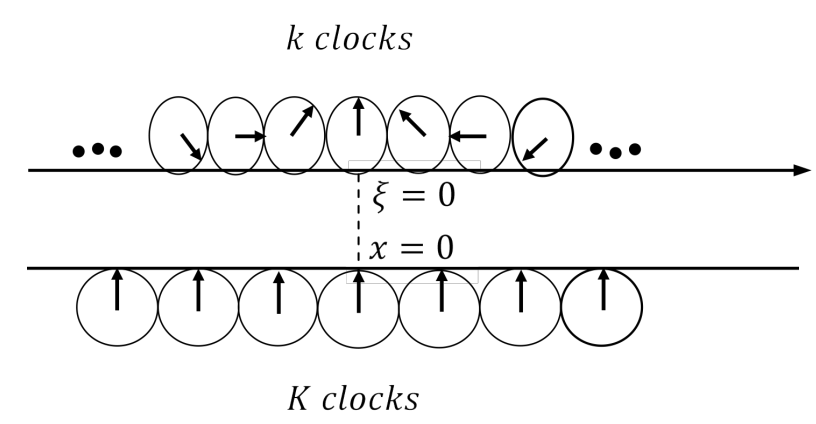

Figure 2. Situation viewed from the "stationary system"

$$
\xi_{\sigma}=\gamma\left[\left(\frac{\sigma}{\gamma^{2}}+\frac{v^{2}}{c^{2}}\right) x_{1}-v t_{1}\right], \tau_{\sigma}=\gamma\left(t_{1}-\frac{v x_{1}}{c^{2}}\right) .
$$

It is evident that all the correspondent events are simultaneous in the $k$ system. Using the formula (2) let us find a distance between two events for two positions $\sigma_{i}<\sigma_{j}$ :

$$
\xi_{j}-\xi_{i}=\gamma\left(\frac{\sigma_{j} x_{1}}{\gamma^{2}}-\frac{\sigma_{i} x_{1}}{\gamma^{2}}\right)=\frac{1}{\gamma}\left(x_{j}-x_{i}\right) .
$$

So, theorem from SRT “distance between two events takes a minimum value in that IRF where they are simultaneous" is hold.

As regard the question of "Only at $x=\xi=0$ do the clocks depicted read the same time in both systems, where $t=\tau=0$ ", let us consider a position depicted in Figure 3. The time $t=T$ is elapsed in the $K$ system, so the origin of the $k$ system $\xi_{0}=0$ is located at the point $x=v T$. Our attention is drawn to the position $x_{n}=\gamma v T /(\gamma+1)$ at this moment $T=t_{n}$. The event $\left(x_{n}, t_{n}\right)$ is subject to the Lorentz transformation (1):

$$
\begin{gathered}
\xi_{n}=\gamma\left(x_{n}-v t_{n}\right)=\gamma\left(\frac{\gamma v T}{\gamma+1}-v T\right)=\gamma v T\left(\frac{\gamma}{\gamma+1}-1\right)=-\frac{\gamma v T}{\gamma+1} ; \\
\tau_{n}=\gamma\left(t_{n}-\frac{v}{c^{2}} x_{n}\right)=\gamma\left(T-\frac{v^{2}}{c^{2}} \frac{\gamma T}{\gamma+1}\right)=\gamma T\left(1-\frac{v^{2}}{c^{2}} \frac{\gamma}{\gamma+1}\right)=\gamma T\left(1-\beta^{2} \frac{\gamma}{\gamma+1}\right) \\
=\gamma T\left(\frac{1-\beta^{2} \gamma+\gamma}{\gamma+1}\right)=\gamma T \frac{1+\gamma\left(1-\beta^{2}\right)}{\gamma+1}=\gamma T \frac{1+1 / \gamma}{\gamma+1}=T \frac{\gamma+1}{\gamma+1}=T .
\end{gathered}
$$

Thus, we have found that always there exists a neutral point where times of both systems $K$ and $k$ coincide: $t_{n}=\tau_{n}=T$. Positions of these neutral points are identical in both systems except of sign: $x_{n}=-\xi_{n}$, i.e. the distances are equal. The Lorentz-Fitzgerald's contraction leads to a fact that the distance $\left[\xi_{n}, \xi_{0}\right]$ viewed from the $K$ system must be divided by relativistic factor:

$$
\left[x_{n}, \xi_{0}\right]=\frac{\left[\xi_{n}, \xi_{0}\right]}{\gamma}=\frac{v T}{\gamma+1} .
$$

The sum is just equal to the full distance between two origins:

$$
\left[x_{0}, x_{n}\right]+\left[x_{n}, \xi_{0}\right]=\frac{\gamma v T}{\gamma+1}+\frac{v T}{\gamma+1}=v T \frac{\gamma+1}{\gamma+1}=v T \text {. }
$$




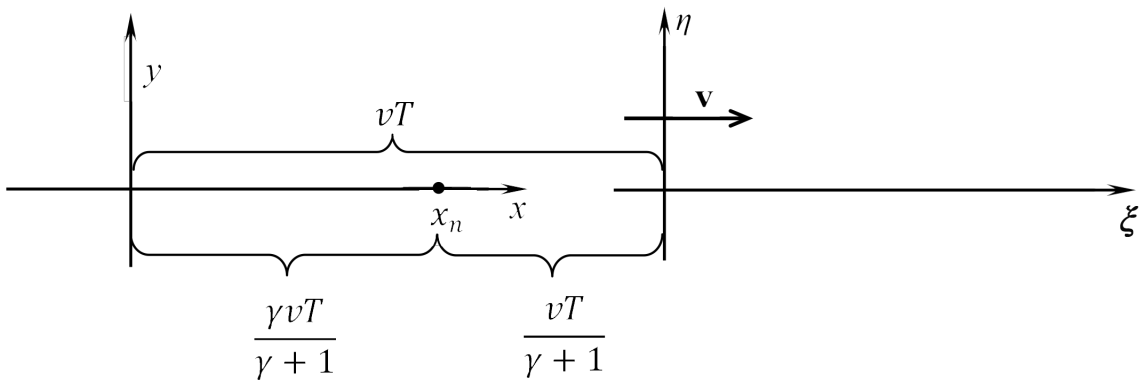

Figure 3. "Neutral" point.

The neutral point is moving along the $x$-axis in positive direction with speed $v_{n}^{x}=v \gamma /(\gamma+1)$. The $K$ system is moving respective to the $k$ system with speed $(-v)$. Using the relativistic rule for vector addition we could find the speed of the neutral point relative to the $k$ system:

$$
v_{n}^{\xi}=\frac{\frac{\gamma v}{\gamma+1}-v}{1-\frac{v^{2}}{c^{2}} \frac{\gamma}{\gamma+1}}=\frac{-v}{\gamma+1-\frac{v^{2}}{c^{2}} \gamma}=\frac{-v}{\gamma+1-\beta^{2} \gamma \pm \gamma}=-\frac{-v}{1+\gamma\left(1-\beta^{2}\right)}=\frac{-\gamma v}{\gamma+1}=-v_{n}^{x} .
$$

No wonder is in the above results, because the principle of relativity asserts the full equivalence of all different inertial reference frames.

\section{About Length Contraction}

In the section 2 the author applies the inverse Lorentz transformation for the special range of events specified in the $k$ system and obtains in the $K$ system some correspondent range of simultaneous events. "Either way, Einstein's system of clock-synchronized stationary observers is inconsistent with the Lorentz Transformation"-states the author. This conclusion is fatally wrong. The very notions "stationary-moving" are quite relative: from the point of view of any $K$-observer the $K$ system is stationary and the $k$ system is in motion, but from the point of view of any $k$-observer the $k$ system is stationary and the $K$ system is the moving one. As a matter of fact, strict qualitative symmetry among all the inertial reference frames (IRF) in the special relativity theory is fundamental and absolute.

Then in the Section 3 the author addresses the procedure of length measurement. There is a thin rigid rod fixed along the abscissa $\xi$ in his own $k$ system. Let $\left(\xi_{1}, \tau_{1}\right)$ and $\left(\xi_{2}, \tau_{2}\right)$ be the simultaneous events $\left(\tau_{1}=\tau_{2}=\tau\right)$ of measurement the locations of its two ends, so that the rod's length is $L_{\xi}=\xi_{2}-\xi_{1}$. The inverse Lorentz transformation gives us these events viewed from the $K$ system:

$$
\left(x_{1}, t_{1}\right)=\left[\gamma\left(\xi_{1}+v \tau\right), \gamma\left(\tau+\frac{v}{c^{2}} \xi_{1}\right)\right] ;\left(x_{2}, t_{2}\right)=\left[\gamma\left(\xi_{2}+v \tau\right), \gamma\left(\tau+\frac{v}{c^{2}} \xi_{2}\right)\right] .
$$

Here the procedure of measurement lost its simultaneity. Thus, the value $\left(x_{2}-x_{1}\right) \neq L_{x}$ because the rod is shifted during the time-interval $\left(t_{2}-t_{1}\right)$ for the distance $\Delta L=v\left(t_{2}-t_{1}\right)$. In this case the real rod's length would be 


$$
L_{x}=\left(x_{2}-x_{1}\right)-\Delta L=\gamma\left(\xi_{2}-\xi_{1}\right)-\gamma \frac{v^{2}}{c^{2}}\left(\xi_{2}-\xi_{1}\right)=\gamma\left(1-\frac{v^{2}}{c^{2}}\right)\left(\xi_{2}-\xi_{1}\right)=\frac{L_{\xi}}{\gamma} .
$$

The rod is contracted by the factor $\gamma$ despite the author's assertion.

In the section 4 the author manipulates with a time-interval. Consider two events occurring in the $K$ system at the same point but at the different instants of time: $\left(x, t_{1}\right)$ and $\left(x, t_{2}\right)$. According to the Lorentz transformation these events in the $k$ system would be $\left(\xi_{1}, \tau_{1}\right)$ and $\left(\xi_{2}, \tau_{2}\right)$, where

$$
\xi_{1}=\gamma\left(x-v t_{1}\right), \tau_{1}=\gamma\left(t_{1}-\frac{v}{c^{2}} x\right) ; \xi_{2}=\gamma\left(x-v t_{2}\right), \tau_{2}=\gamma\left(t_{2}-\frac{v}{c^{2}} x\right) .
$$

Let us find the ratio between two time-intervals existing in the different systems:

$$
\Delta \tau=\tau_{2}-\tau_{1}=\gamma\left(t_{2}-t_{1}\right)=\gamma \Delta t
$$

So, second theorem from SRT "time-interval between two events takes a minimum value in that IRF where these events occur at the same location" is hold, in contrast to the inference made by the author.

\section{Conclusions}

The further analysis of the article would be senseless because it just seems to criticize the special relativity theory. The author neglects basic tenets of the SRT, foists his own and confuses this makeshift "theory" with Einstein's creature. Similar papers appear from time to time in scientific journals, for example, works [3] [4] by K. Suto, who intented classical common sense to be opposed to the strictly elaborated theory and took the lawful relativity of simultaneity for an inadmissible discrepancy. In the paper [5] Suto found a would-be contradiction in the SRT when based his arguing on the assertion: "According to the SRT a stationary observer finds the following relationship between his own time $t$ and the time $t^{\prime}$ which elapses on a moving clock: $t^{\prime}=t / \gamma$ (2)". But, as we could be convinced of, this ratio is valid in a specific position only. Using (1) for any concrete moment $t=T$, we readily obtain $\tau=T / \gamma$ at the position $x=v T$, and side by side with that we obtain opposed $T=\tau / \gamma$ at the position $t=0$. In the SRT all inertial frames of reference are tantamount one to another, and all observers have complete equality of rights. An observer in his own IRF' could assert with the same success that, quite the contrary, $t=t^{\prime} / \gamma$. So, the above-mentioned statement by Mr. Suto proves to be senseless, away with confusing reference to the "clock paradox", where three IRFs appear on the scene!

In his thought experiment the author uses "light signal emitted from the two light sources at the instant that $O$ and $O$ pass by each other" and concludes "Now, are the two inertial systems truly equivalent, as claimed by the STR?" Let us consider a situation some time later the start (Figure 4). In the $k$ system there is an event $\left(c t^{\prime}, t^{\prime}\right)$ when light emitted $t^{\prime}$ seconds ago from the coordinate origin $O$ reaches the point with a coordinate $\xi=c t^{\prime}$. The Lorentz transformation gives the following components of this event in the $K$ system: 


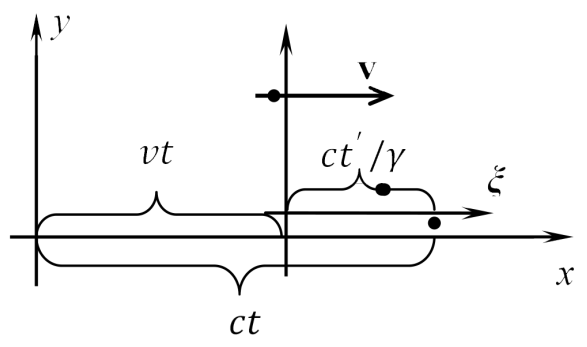

Figure 4. Light signals.

$$
\begin{aligned}
& x=\gamma\left(c t^{\prime}+v t^{\prime}\right)=\gamma c t^{\prime}\left(1+\frac{v}{c}\right) \\
& t=\gamma\left(t^{\prime}+\frac{v}{c^{2}} c t^{\prime}\right)=\gamma t^{\prime}\left(1+\frac{v}{c}\right)
\end{aligned}
$$

Due to the Lorentz-Fitzgerald's length contraction the distance $c t^{\prime}$ is shorten to the value $c t^{\prime} / \gamma$ in the $K$ system and we could check the distance balance. From the Equation (3) we have

$$
t^{\prime}=\frac{t}{\gamma\left(1+\frac{v}{c}\right)},
$$

Hence,

$$
\begin{aligned}
v t+\frac{c t^{\prime}}{\gamma} & =v t+\frac{c t}{\gamma^{2}\left(1+\frac{v}{c}\right)}=v t+\frac{c t\left(1-\frac{v}{c}\right)\left(1+\frac{v}{c}\right)}{\left(1+\frac{v}{c}\right)} \\
& =v t+c t\left(1-\frac{v}{c}\right)=c t\left(\frac{v}{c}+1-\frac{v}{c}\right)=c t .
\end{aligned}
$$

Thus, the light signal turns to be located at the same point seen either from the $k$ system or from the $K$ system. No discrepancy or contradiction is present, and both systems are truly (despite the question-mark occurring at the end of above citation) equivalent in the realm of the SRT.

Three authors of the paper [6] suggest five "new paradoxes" discovered in the SRT, however, all of them proved to be pseudo-paradoxes provided by arbitrary treatment of the relativistic axioms (see [7]). There is nothing as ludicrous as an unsubstantiated criticism. As regard the question of an authentic critical approach, real (not imaginary!) inconsistencies indwelling to the special relativity theory are pointed out and critics of in the papers [8] [9] [10] [11].

\section{References}

[1] Crother, S.J. (2017) On the Logical Inconsistency of the Special Theory of Relativity. American Journal of Modern Physics, 6, 43-48. https://doi.org/10.11648/j.ajmp.20170603.12

[2] French, A.P. (1981) Special Relativity. Thomas Nelson \& Sons, Great Britain.

[3] Suto, K. (2016) Elucidation of Time Symmetry Predicted by the Special Theory of 
Relativity. IOSR Journal of Applied Physics, 8, 20-27.

[4] Suto, K. (2017) An Elucidation of the Symmetry of Length Contraction Predicted by the Special Theory of Relativity. Applied Physics Research, 9, 31-39.

https://doi.org/10.5539/apr.v9n3p31

[5] Suto, K. (2016) Thought Experiment Revealing a Contraction in the Special Theory of Relativity. Applied Physics Research, 8, 70-74. https://doi.org/10.5539/apr.v8n6p70

[6] Artekha, S., Chubykalo, A. and Espinoza, A. (2016) Some of the Complexities in the Special Relativity: New Paradoxes. Physical Science International Journal, 11, 1-15. https://doi.org/10.9734/PSIJ/2016/26788

[7] Leus, V.A. (2016) Critical Comments on the Paper "Some of the Complexities in the Special Relativity: New Paradoxes. Physical Science International Journal, 12, 1-5.

[8] Leus, V.A. (2015) Triplet Paradox in Special Relativity and Discrepancy with Electromagnetism. American Journal of Modern Physics (Special issue: Physics of Time: Theory and Experiment), 4, 26-33.

https://doi.org/10.11648/j.ajmp.s.2015040201.15

[9] Wagner, D. (2015) Fresnel versus Einstein: A New Direct Test. Applied Physics Research, 7, 33-40. https://doi.org/10.5539/apr.v7n3p33

[10] Leus, V.A. (2015) On an Optical Paradox: Theory and Experiment. Applied Physics Research, 7, 20-25. https://doi.org/10.5539/apr.v7n6p20

[11] Leus, V.A. (2018) Relativistic Paradox Characteristic for Belt Transmission with the Bell-Effect. Journal of Modern Physics (Special Issue "Wortmhole").

https://doi.org/10.4236/jmp.2018.95066 